\title{
Analytical Study for the Charge-Transfer Complexes of Risperidone in Pure and Dosage Forms
}

\author{
F. Ibrahim, N. El-Enany, Sh. Shalan and R. El-Sharawy.
}

Department of analytical chemistry, Faculty of pharmacy, University of Mansoura, 35516, Mansoura, Egypt E-mail: rasha.elsharawy@yahoo.com

\section{ABSTRACT}

Two simple, accurate and sensitive spectrophotometric methods were carried out to investigate through charge-transfer reactions of risperidone (RIS) as n-electron donor with various $\pi$ acceptors: 7, 7, 8, 8-tetracyanoquinodimethane (TCNQ) and $\mathrm{p}$-chloranilic acid (pCA). The absorbance of reaction product was measured at 842 and $520 \mathrm{~nm}$ for TCNQ and pCA reagents respectively. Different experimental parameters affecting the reactions were carefully studied. The reaction pathway was postulated. The proposed spectrophotometric method was utilized for the analysis of RIS in pure form as well as in its pharmaceutical preparations. Under the optimum reaction conditions, Beer's law is obeyed over the concentration range of $1-12 \mu \mathrm{g} \mathrm{mL}^{-1}$ and $10-180 \mu \mathrm{g} \mathrm{mL}^{-1}$ for TCNQ and $\mathrm{pCA}$ respectively. The limit of assays detection (LOD) is $0.114 \mu \mathrm{g} \mathrm{mL}^{-1}$ and $2.55 \mu \mathrm{g} \mathrm{mL}^{-1}$ for TCNQ and pCA respectively. The mean recovery percentage was $99.72 \pm$ 1.06 and $100.50 \pm 1.07$ for TCNQ and $\mathrm{pCA}$ respectively. The results were compared favorably with those obtained by comparison method. The proposed method was validated statistically according to ICH guidelines.

\section{KEYWORDS}

spectrophotometry; charge transfer; Risperidone; p-chloranilic acid and 7.7.8.8 tetracyanoquinodimethane.

\section{Council for Innovative Research}

Peer Review Research Publishing System

Journal: Journal of Advances in Chemistry

Vol. 8, No. 3

editor@cirjac.com

www.jac.cirworld.com, member.cirworld.com 


\section{INTRODUCTION}

Risperidone (RIS) is belonging to the chemical class of Benzisoxazole derivatives and chemically is 4-(2-(4-(6Flurobenzo[d]isoxazd-3yl]1-piperidyl]ethyl]-3-methyl-2,6 diazabicyclodeca-1,3-dien-5-one [1] with molecular formula $\mathrm{C}_{23}$ $\mathrm{H}_{27} \mathrm{FN}_{4} \mathrm{O}_{2}$ was presented in (Fig. 1) RIS is an antipsychotic agent [2], which acts through selective antagonism of serotonin 5HT2, dopamine D2 receptors, used in the treatment of schizophrenia and other psychoses [3]. It is mostly metabolized by alicyclic hydroxylation and oxidative $\mathrm{N}$ - dealkylation [4]. An ideal stability indicating method is one that quantifies the drug and also resolves its degradation products [5]. Literature review for RIS analysis revealed several methods based on different technique such as HPLC with UV detection [6].Visible spectrophotometric methods [7], LC-ms and HPLC ESI/MS assay for its quantification in plasma and serum [8-11], Chiral Chromatography [12], Pulse Polarography [13], Chemiluminescence assay [14], LC with coulometric Detection [15].

Since the other reported methods are either tedious [6] or time consuming [8-15], this encourage us to develop simple, sensitive and specific spectrophotometric methods for the determination of RIS in bulk drug and different pharmaceutical formulations.

The proposed methods are based on the charge transfer complexation reaction of RIS with either 7, 7, 8, 8tetracyanoquinodimethane (TCNQ) or $\mathrm{p}$ - chloranilic acid $(\mathrm{pCA})$ respectively without prior extraction step. The proposed methods were validated statistically.

\section{EXPERIMENTAL}

\subsection{Apparatus}

UV-1601PC (Shimadzu, Japan) ultraviolet-visible spectrophotometers with matched 1-cm matched quartz cells, and spectro UV, Vis double beam pc scanning spectrophotometer uvd, 2950.

\subsection{Materials and reagents}

Risperidone was used as working standard. The standard solution was prepared by dissolving $10 \mathrm{mg}$ of the drug in 100 $\mathrm{mL}$ of methanol in case of TCNQ and $100 \mathrm{mg}$ of the drug in $100 \mathrm{~mL}$ of acetonitrile in case of $\mathrm{pCA}$, respectively.

All other reagents used were of Analytical Reagent grade. Solutions of TCNQ was $(0.1 \%)$ in acetonitrile and the solution was stable for at least 1 week at $4{ }^{\circ} \mathrm{C}$ and pCA was $(0.3 \%)$ in acetonitrile, and the solution was freshly prepared.

\subsection{Pharmaceutical preparation}

Sigmadone ${ }^{\circledR}$ tablets product of (SIGMA pharmaceutical industries, Egypt, S.A.E.), labeled to contain $3 \mathrm{mg}$ RIS per tablet, with batch $\neq 00846$.

Riscure $4^{\circledR}$ tablets product of (GLOBAL NAPI PHARMACEUTICALS (GNP), Egypt), labeled to contain $4 \mathrm{mg}$ RIS per tablet, with batch $\neq 112521 \mathrm{~A}$.

Psychodal $^{\circledR}$ solution (DELTA PHARMA S.A.E., Egypt), each $\mathrm{mL}$ contains $1 \mathrm{mg}$ RIS, with batch $\neq 11492$.

All pharmaceutical preparations were obtained from local pharmacy.

\subsection{Procedure for determination}

\subsubsection{TCNQ method}

Aliquots of RIS $(0.01 \%)$ corresponding to $10 \mu \mathrm{g} \mathrm{mL}^{-1}$ with final working range $\left(1-12 \mu \mathrm{g} \mathrm{mL} \mathrm{L}^{-1}\right)$ were transferred to a serious of $10 \mathrm{ml}$ volumetric flasks. To each flask, $1.5 \mathrm{~mL}$ of $(0.1 \%) \mathrm{TCNQ}$ was added and heated at $90^{\circ} \mathrm{C}$ for $5 \mathrm{~min}$ then completed to volume with acetonitrile. The absorbance of the colored product was measured at $842 \mathrm{~nm}$ against reagent blank prepared simultaneously. Calibration curve was obtained by plotting the concentration of the drug versus absorbance; alternatively corresponding regression equation was derived.

\subsubsection{PCA method}

Different aliquots of RIS $(0.1 \%)$ corresponding to $100 \mu \mathrm{g} \mathrm{mL}^{-1}$ with final working range $\left(10-180 \mu \mathrm{g} \mathrm{mL}^{-1}\right)$ were transferred to a serious of 10 volumetric flasks. To each flask, $4 \mathrm{~mL}$ of $(0.3 \%) \mathrm{pCA}$ was added and complete to volume with acetonitrile. The absorbance of the colored product was measured at $520 \mathrm{~nm}$ against reagent blank prepared simultaneously. Calibration curve was obtained by plotting the concentration of the drug versus absorbance; alternatively corresponding regression equation was derived.

\subsection{Analysis of pharmaceutical formulations}

\subsubsection{Preparation of tablets sample}

Ten tablets of each formulation were weighed and finely pulverized and mixed well. A quantity of the powder equivalent to $10 \mathrm{mg}$ and $100 \mathrm{mg}$ was transferred into a $100 \mathrm{~mL}$ conical flask and extracted with $3 \times 30 \mathrm{~mL}$ of either methanol or acetonitrile for TCNQ or PCA respectively via sonicator for $5 \mathrm{~min}$, filtered into $100 \mathrm{~mL}$ measuring flask and completed to volume with the corresponding solvent then shaked well. 
A measured volume of the filtrate was diluted quantitatively with a suitable conc. lies in the linear range of each particular assay method.

Then the procedures described under section 2.4.1 and 2.4.2 was performed. The nominal content of the drug was determined from the corresponding calibration curve or regression equation.

\subsubsection{Preparation of oral solution sample}

Aliquots of Psycodal oral solution $(1 \mathrm{mg} / \mathrm{mL})$ were taken and dissolved in $10 \mathrm{~mL}$ chloroform and shacked for $10 \mathrm{~min}$, and then the chloroform layer was separated and evaporated on water bath till dryness. The residue was reconstituted in acetonitrile and the absorbance was measured at the specific wavelength. Then the procedures described under section 2.4.1 and 2.4.2 was performed. The nominal content of the drug was determined from the corresponding calibration curve or regression equation.

\subsection{Determination of molar ratio}

The Job's method of continuous variation was employed [16]. Standard solutions of RIS and reagents were prepared. The concentrations of these solutions were $0.5 \times 10^{-3} \mathrm{M}$ (in methanol for TCNQ), $5 \times 10^{-3} \mathrm{M}$ (in acetonitrile for $\mathrm{pCA}$ ). The reactions were allowed to proceed for the optimum reaction time and then the absorbance of the resulting solutions was measured at the corresponding wavelengths of maximum absorbance ( $\lambda$ max). Fig (2)

\section{RESULTS AND DISSCOSSION}

Different experimental parameters affecting development and stability of reaction product were carefully studied while others were kept constant. These parameters include; effect of reaction time, temperature, solvent, and volume of the reagent.

\subsection{Spectral characteristics of the reaction (reaction with $\pi$ receptors)}

In polar solvents such as methanol or acetonitrile, complete electron transfer from the RIS (D), as an electron donor, to the acceptor moiety $(A)$ takes place with the formation intensely colored radical ions with high molar absorptivity values, according to the following scheme:

$$
\begin{aligned}
& D+A \leftrightarrow(D-A) \leftrightarrow D^{+}+A^{-} \\
& \text {Complex Radical ions }
\end{aligned}
$$

the dissociation of the (D-A)complex was promoted by the high ionizing power of the polar solvents and the resulting peaks in the absorption spectra of RIS- acceptor reaction mixtures.

The interaction of RIS with $\pi$-acceptors at $90 \circ \mathrm{C}$ and at room temperature produced colored chromogenes exhibiting maximum absorption at 842, $520 \mathrm{~nm}$ for TCNQ and pCA, respectively. Fig (3)

The predominant chromogen with TCNQ in acetonitrile is the green colored anion, which exhibits maxima peaking at 842 and $762 \mathrm{~nm}$. These bands may be attributed to the formation of the radical anion TCNQ•, which was probably formed by the dissociation of an original donor-acceptor (D-A) complex with the RIS. The dissociation of the complex was promoted by the high ionizing power of acetonitrile.

Chloranilic acid (pCA) exists in three ionic forms, the neutral yellow-orange $\mathrm{H}_{2} \mathrm{~A}$ at very low $\mathrm{pH}$, the dark purple $\mathrm{HA}$ which is stable at high $\mathrm{pH}$; these transformations are illustrated in the following scheme:

$$
\begin{aligned}
& \mathrm{H}_{2} \mathrm{~A} \leftrightarrow \mathrm{H}^{+}+\mathrm{HA}^{-} \text {(violet), } \\
& \mathrm{HA}^{-} \leftrightarrow \mathrm{H}^{+}+\mathrm{A}^{2-} \text { (colorless) }
\end{aligned}
$$

Since the interaction of RIS with PCA in acetonitrile gave red-orange product, it might be concluded that $\mathrm{HA}^{-}$was the form of pCA involved in the reaction described herein.

\subsection{Effect of the reaction time and temperature}

The effect of time on the complexation reactions at room temperature was examined. It was observed that the maximum and stable absorbance of RIS-TCNQ was achieved after heating at $90^{\circ} \mathrm{C}$ for 5 min., whereas maximum and stable absorbance for RIS-pCA was obtained at once at room temperature.

\subsection{Effect of solvent}

The effect of solvents such as acetonitrile, acetone and methanol on the absorbance of reaction product was studied. Experimental results indicated that acetonitrile was the solvent of choice for TCNQ and pCA. Methanol was the solvent of choice for RIS in case RIS-TCNQ complex and acetonitrile was the solvent of choice in case of RIS-pCA complex regarding stability and maximum absorption of the reaction product. 


\subsection{Effect of reagent volume and concentration}

The optimum conditions for the TCNQ and pCA methods were established by varying the volume of reagent and keeping the drug concentration constant.

To establish the optimum experimental condition for RIS-TCNQ charge transfer complex, the drug was allowed to react with varying volumes of $(0.1 \%) \mathrm{TCNQ}$. The maximum absorbance was achieved with $1.5 \mathrm{~mL}$ and further increase in the volume resulted in a gradual decrease in the absorbance value. Therefore a volume of $1.5 \mathrm{~mL}$ was used as an optimum volume.

Regarding pCA method, it was found that increasing in the volume of $(0.3 \%)$ pCA resulted in a gradual increase in the absorbance of the reaction product up to $4 \mathrm{~mL}$ and after which the absorbance of the reaction product remained constant, therefore, $4 \mathrm{~mL}$ of pCA was chosen as the optimum volume of the reagent.

\subsection{Development and validation of the analytical methods}

The validity of the proposed method was tested regarding linearity, specificity, accuracy, repeatability and precision according to ICH Q2B recommendations [17].

\subsubsection{Linearity}

Using the above procedure, a linear regression equation was obtained. The regression plot showed that there was a linear dependence of the absorbance on the concentration of the drug. Linear regression analysis of the data gave the following equation:

$$
\begin{array}{lll}
A=-0.0547+0.088 C & (r=0.9999) & \text { for TCNQ } \\
A=0.0205+0.0047 C & (r=0.9999) & \text { for } \mathrm{pCA}
\end{array}
$$

Where the $A$ is the absorbance, $C$ is the concentration of the drug in $\mu \mathrm{g} \mathrm{mL}^{-1}$ and $r$ is the correlation coefficient. The limit of quantitation (LOQ) and limit of detection (LOD) were calculated according to ICH Q2B and results are shown in (table 1). The values of LOQ and LOD were calculated according to the following equation (17)

$$
\mathrm{LOQ}=10 \text { (S.D. } a / b) ; \quad \mathrm{LOD}=3.3 \text { (S.D. } a / b)
$$

Where $a$ is intercept of regression line and $b$ is the slope of the calibration curve.

The proposed method was evaluated for accuracy as percentage relative error (\% error) and the precision as percentage relative standard deviation (\% RSD). (Table 1)

\subsubsection{Accuracy}

To test the validity of the proposed method it was applied to the determination of pure samples of RIS over the working concentration range. The results obtained were in a good agreement with those obtained using comparison method [20]. Using student t-test and variance F-test revealed no significant difference between the performance of the two methods regarding the accuracy and precision, respectively [21]. (Table 3)

The comparison method was based on preparation of the stock by transfer $100 \mathrm{mg}$ to $100 \mathrm{~mL}$ volumetric flask and complete to the volume with methanol and measure the absorbance directly. The absorbance of the reaction product was measured at 240 and $280 \mathrm{~nm}$ and the concentration was rectilinear over the range of 20 to $60 \mu \mathrm{gL}^{-1}$ [20].

The validity of the method was proven by statistical evaluation of the regression lines regarding the standard deviation of the residuals $\left(S_{y / x}\right)$, the standard deviation of the intercept $\left(S_{a}\right)$ and the standard deviation of the slope $\left(S_{b}\right)$. The results are summarized in (table 1).

The small values for these figures point to low degree of scattering of the points around the calibration line and high precision.

\subsubsection{Precision}

The repeatability was determined by applying the proposed method for the determination of three concentrations of RIS in the pure form three successive times, and the results are listed in (table 2). Low percentage error and low percentage RSD indicate high accuracy and high precision of the proposed method [18-19].

\subsubsection{Robustness}

The robustness of the method was assessed by evaluating the influence of small variation of experimental variables: concentrations of acceptor reagent, and reaction time, on the analytical performance of the method. In these experiments, one experimental parameter was changed while the other parameters were kept unchanged, and the recovery percentage was calculated each time. The small variations in any of the variables did not significantly affect the results; recovery percentage in case of TCNQ is $99.73 \pm 0.90$ and in case of pCA is $100.50 \pm 1.07$. This provided an indication for the reliability of the proposed methods during routine work. 


\subsection{Application of the proposed method to the analysis of the pharmaceutical preparations}

The proposed method was applied to the determination of RIS in pharmaceutical preparations. The method was tested for linearity, specifity, accuracy, repeatability and precision according to ICH Q2B recommendations [17].

\subsubsection{Selectivity}

The selectivity of the method was investigated by observing any interference encountered from the common tablet excipients. These excipients did not interfere with the proposed method.

\subsubsection{Accuracy}

The results of the proposed method were statistically compared with those obtained using the comparison method [20]. Statistical analysis of the results, using Student $t$-test and variance ratio F-test revealed no significant difference between the performance of the proposed and the comparison method regarding the accuracy and precision.

\subsection{Application of the method to the analysis of tablets}

The obtained satisfactory validation results made the proposed procedures suitable for the routine quality control analysis of RIS. The proposed and the reported methods were applied to the determination of RIS in its tablets. The results obtained by the proposed methods were statistically compared with those obtained by the comparison method at which

In the t- and F-tests, no significant differences were found between the calculated and theoretical values of both the proposed and the reported methods at 95\% confidence level [21]. This indicated similar precision and accuracy in the analysis of RIS in its tablets. It is evident from these results that all the proposed methods are applicable to the analysis of RIS in its tablets with comparable analytical performance. However, the critical recommendations of some of these methods might be based on the experimental conditions (e.g. reaction time), and the ultimate sensitivity that determines the amount of specimen required for analysis. For example, the method involving pCA is recommended whenever rapid analysis is required; this because they have very short reaction time. The method involving TCNQ is recommended, as high sensitivity is required on the expense of the analysis time.

\section{CONCLOSION}

The charge-transfer complexation reaction of risperidone (RIS) as electron donor and some electron acceptors has been investigated. The obtained complexes were studied by ultraviolet-visible spectrophotometry technique. The obtained colored complexes were utilized in the development of two simple, rapid and accurate spectrophotometric methods for the analysis of RIS in pure form as well as in tablets and oral solution.

Table 1: Analytical performance data for the proposed method.

\begin{tabular}{|l|c|c|}
\hline Parameters & TCNQ & pCA \\
\hline Linearity range $\left(\mu \mathrm{g} \mathrm{mL}^{-1}\right)$ & $1-12$ & 2.5548 \\
\hline Limit of detection (LOD) $\mu \mathrm{g} \mathrm{mL}^{-1}$ & 0.0999 & 7.7417 \\
\hline Limit of quantification(LOQ) $\mu \mathrm{g} \mathrm{mL}^{-1}$ & 0.3027 & 0.0205 \\
\hline Intercept (a) & -0.0547 & 0.0047 \\
\hline Slope (b) & 0.0881 & 0.9999 \\
\hline Correlation coefficient & 0.9999 & 0.0054 \\
\hline Standard deviation of residual $S_{(y / x)}$ & 0.0038 & 0.0036 \\
\hline Standard deviation of intercept $\left(S_{a}\right)$ & 0.0027 & 0.0000 \\
\hline Standard deviation of slope $\left(S_{b}\right)$ & & 1.06 \\
\hline \% RSD & 0.0004 & 0.38 \\
\hline
\end{tabular}


Table 4: Application of the proposed method to the pharmaceutical preparation using TCNQ.

\begin{tabular}{|c|c|c|c|c|}
\hline Parameters & \multicolumn{2}{|c|}{ Riscure ( 4 mg) ${ }^{\text {(a) }}$} & \multicolumn{2}{|c|}{ Sigmadone $(3 \mathrm{mg})^{(b)}$} \\
\hline \multirow{4}{*}{ \%Recovery } & Proposed method & Comparison method & Proposed method & Comparison method \\
\hline & 98.81 & 99.00 & 100.71 & 99.00 \\
\hline & 100.29 & 101.00 & 101.95 & 101.00 \\
\hline & 99.21 & 99.67 & 99.63 & 99.67 \\
\hline Mean & 99.44 & 99.89 & 100.76 & 99.89 \\
\hline \pm S.D. & 0.77 & 1.02 & 1.16 & 1.02 \\
\hline Student t- test & \multicolumn{2}{|c|}{$0.61(2.132)^{*}$} & \multicolumn{2}{|c|}{$0.98(2.132)^{*}$} \\
\hline $\begin{array}{c}\text { Variance ratio } \\
\text { F-ratio }\end{array}$ & \multicolumn{2}{|c|}{$1.77(19)^{*}$} & \multicolumn{2}{|c|}{$1.3(19)^{*}$} \\
\hline
\end{tabular}

Table 5: Application of the proposed method to the pharmaceutical preparation using pCA.

\begin{tabular}{|c|c|c|c|c|}
\hline Parameters & \multicolumn{2}{|c|}{ Tablets } & \multicolumn{2}{|c|}{ Oral solution } \\
\hline & \multicolumn{2}{|c|}{ Sigmadone (3 mg) } & \multicolumn{2}{|c|}{ Psychodal $^{\text {(c) }}$} \\
\hline & $\begin{array}{l}\text { Proposed } \\
\text { method }\end{array}$ & Comparison method & $\begin{array}{l}\text { Proposed } \\
\text { method }\end{array}$ & $\begin{array}{c}\text { Comparison } \\
\text { method }\end{array}$ \\
\hline \multirow{3}{*}{$\%$ Recovery } & 101.22 & 99.0 & 101.06 & 99.0 \\
\hline & 101.33 & 101.00 & 98.40 & 101.00 \\
\hline & 100.12 & 99.67 & 100 & 99.67 \\
\hline Mean & 100.89 & 99.89 & 99.82 & 99.89 \\
\hline \pm S.D. & 0.67 & 1.02 & 1.34 & 1.02 \\
\hline Student t- test & \multicolumn{2}{|c|}{$1.28(2.132)^{*}$} & \multicolumn{2}{|c|}{$0.187(2.132)^{*}$} \\
\hline Variance ratio F-ratio & \multicolumn{2}{|c|}{$2.23(19)^{*}$} & \multicolumn{2}{|c|}{$1.79(19)^{*}$} \\
\hline
\end{tabular}

* Values between ( ) are the tabulated $t$ and $f$ values respectively at $P=0.05$ [21].

Tablets: $\quad$ (a) Riscure ${ }^{\circledR} 4$ mg product of (GLOBAL NAPI PHARMACEUTICALS (GNP), Egypt), with batch $\neq 112521 \mathrm{~A}$.

(b) Sigmadone ${ }^{\circledR} 3 \mathrm{mg}$ product of (SIGMA pharmaceutical industries, Egypt, S.A.E.), with batch $\neq 00846$.

Oral solution: (c) Psychodal ${ }^{\circledR}$ (DELTA PHARMA S.A.E., Egypt), each $\mathrm{mL}$ contains $1 \mathrm{mg}$ RIS, with batch $\neq 11492$. 


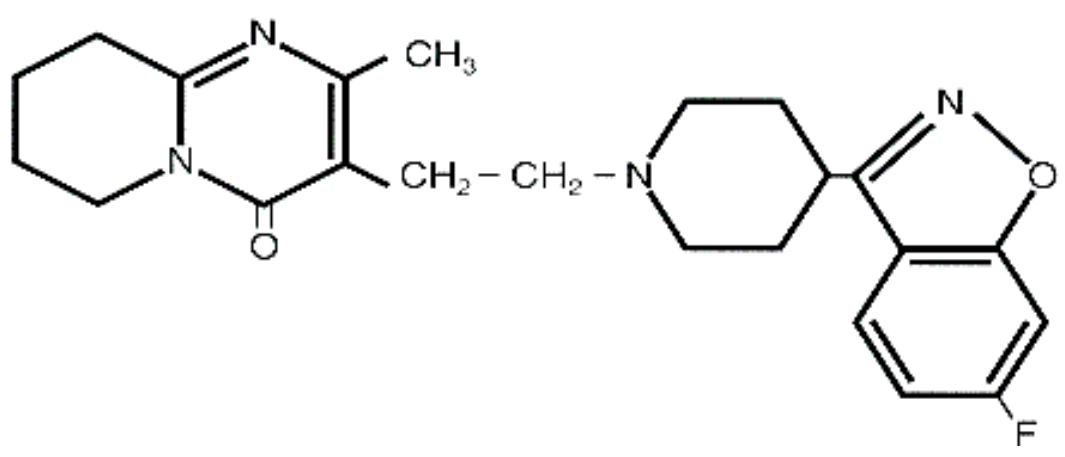

Figure (1): Structure formula of Risperidone

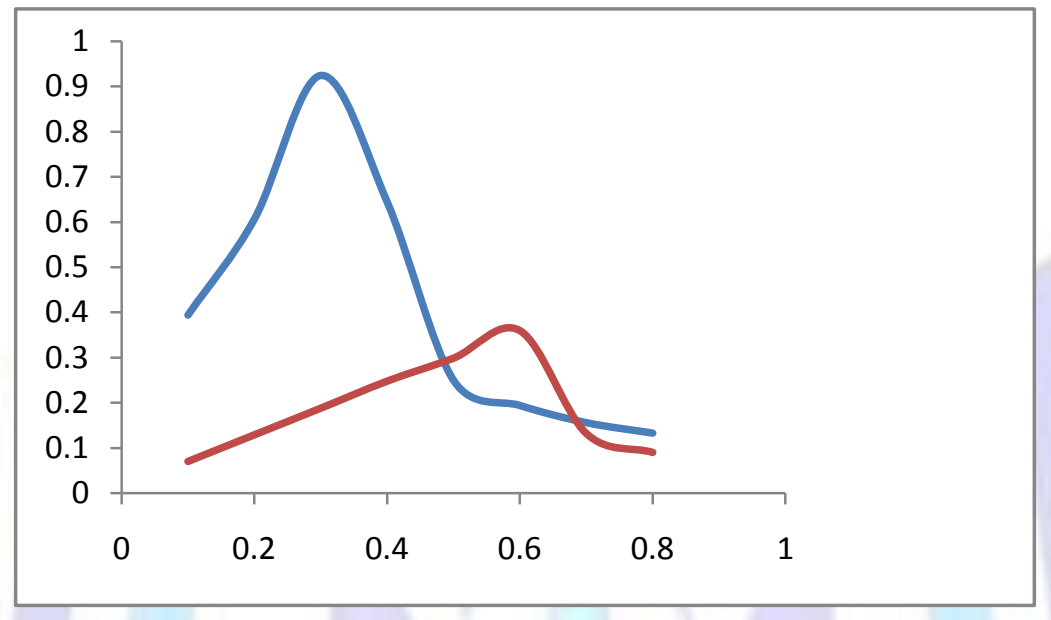

Figure (2): job's plot for RIS-TCNQ complex (abs1) and RIS-pCA complex (abs2).

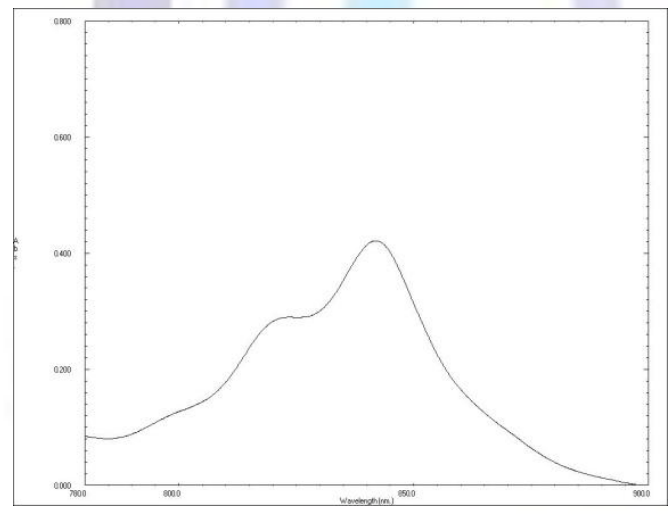

(a)

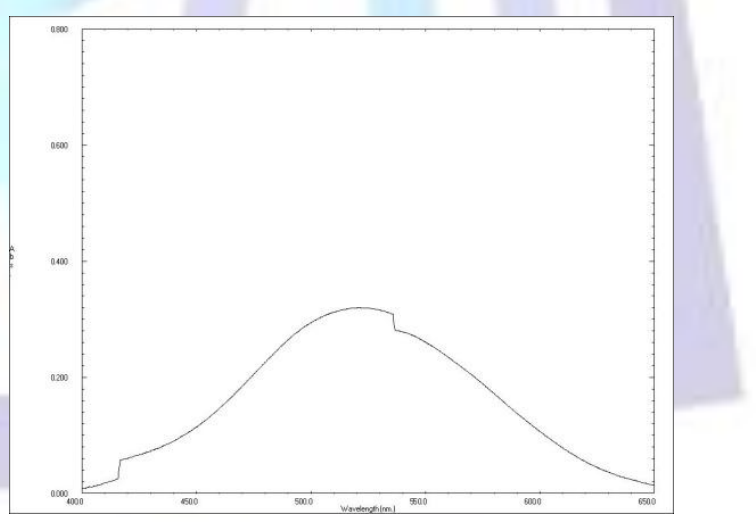

(b)

Figure (3): absorption spectra of TCNQ (a) and pCA (b) with RIS.

\section{REFERENCES}

[1] The Merck Index, Merck Research Laboratories division of Merck and company, 13th ed, NJ, USA, $2001,1627$.

[2] British Pharmacopoeis, Vol.I, 4th Edn., 2002, 1,1500.

[3] Hardman, G., Limbid, L.E. and Gilman, 2001, A.G., the Pharmacological Basis of Therapeutics, McGraw Hill. 10th Edn. ,279.

[4] Tripathi K.D., 1996, Essentials of Medical Pharmacology, 5th ed, Jaypee Brothers, Medical publishers, New Delhi, $747,150,391,394,396,397$. 
[5] ICH, QIA Stability Testing of New Drug Substances and Products, Int Conf on Harmonization, Geresa, November.

[6] Baldaniya S.L., Bhatt K. K., Mehta R. S., Shah D. A., , 2008, "RP-HPLC estimation of RIS in tablet dosage forms" Indian J. of Pharm. Sci., 70 (4), 494- 497.

[7] Singhvi I, Goyal A, "Visible spectrophotometric determination of RIS in tablet formulations "accessed on www.pharmainfo.net on 25/04/08.

[8] Huang MZ, Shentu J. Z., Chen J. C., Liu J. and Zhou H, , 2008, "Determination of RIS in human plasma by HPLC-MS and its application to a pharmacokinetic study in Chinese volunteer's" J Zhejiang Univ. Sci B., 9(2), 114-120.

[9] Zhou Z., L. xin, Kunyan L., Zhihong X., Zeneng C., Wenxin P., Wang F., Zhu R. and Huande L., 2004, "Simultaneous determination of clozapine, olanzapine, RIS and quetiapine in plasma by high-performance liquid chromatographyelectrospray ionization mass spectrometry" journal of Chromatography B, 802 (2), 257-262.

[10] Bartlett M.G., Zhang G. and Terry Jr.A V, 2007, "Simultaneous determination of five antipsychotic drugs in rat plasma by high performance liquid chromatography" journal of Chromatography B, 856(1-2), 20-28

[11] Subbaiah G., Singh S. and Bhatt J., 2006, "Liquid chromatography/tandem mass spectrometry method for simultaneous determination of RIS and its active metabolite 9-hydroxyRES in human plasma" Rapid Communications in Mass Spectrometry, 20(14), 2109-2114.

[12] Danel C., Barthelemy C., Azarzar D., Robert H., Bonte J. P., Odou P. and Vaccher C., 2007, "Analytical and semipreparative enantioseperation of 9- hydroxyRIS, the main metabolite of RIS, using high-performance liquid chromatography and capillary electrophoresis. Validation and determination of enatiomeric purity", J. Chromatogr A., , 1163(1-2), 228-36/

[13] Joshi A., Jeyaseelan C. and Jugade R., 2006, "Differential pulse polarographic studies of RIS in pharmaceutical formulations", Croat. Chem. Acta, 79(4), 541-544.

[14] Song Z. and Wang C., 2004, "Sensitive chemiluminescence assay for RIS in pharmaceutical preparations", J. Pharm.Biomed. Anal., 36(3), 491-494.

[15] Schatz D. S. and Saria A., 2000, "Simultaneous Determination of Paroxetine, RIS and 9-HydroxyRIS in Human Plasma by High-Performance Liquid Chromatography with Coulometric Detection" Phrmacology, (60), 51 - 56.

[16] P. Job, Ann. Chem. 16 (1936) 97.

[17] US Departement of Health and Human Services, Food and Drug Administration, Center for Drug Evaluation and Research. Guidance for Industry Bioanalytical Method Validation. Rockville, MD, May 2001. Available from:

http://www.fda.gov/eder/guidance/4252fnl.pdf(accessed 1 September 2004)

[18] F.W. Fifield and D. Kealey, 2000 , 5th ed. , Black Well Science Ltd. , 270-276.

[19] G.C. Hokanson , 1994 , A Life Cycle Approach to the Validation of Analytical Method During Pharmaceutical Product Development, Part 2 : Changes and the Need for Additional Validation, Pharm. Tech. , 92-100.

[20] G.Alagumani Vasagam, A. Anton Smith, CH. Nawas, M. Sravan Kumar, A. Kattai Muthu and R. Manavalan, 2010 , " Development of Analytival Method for Risperidone by UV Spectrophotometry Using Methanol as a Solvent", Scolars Research Library "Der pharma chemica”, 2 (3) , 309-315.

[21] N.Erk, (2001) J. Pharm. Biomed. Anal. 24, 603-611. 